\title{
Ego sentio, ergō sum!
}

\section{/Introductory part/}

The article offered to your attention is, in substance, a series of conceptually connected excerpts from authorial treatise on the psychological background of pre-cognitive brain activism and some little-known hidden features of the functioning of the higher nervous system. Well and besides, a couple of chapters are devoted here to the innate physiological asymmetry of the hemispheres, and also to the denial of the relevance of true free will to humans.

But in general, by and large, in his scientific views the author tries to adhere to the idea of the subjective reality of emotions and the relative illusory nature of sensations. In its spirit, this position is most likely close to Lenin's than to the earlier - the German classical one (the founder is $\mathrm{H}$. von Helmholtz). The latter, we recall, exactly one and a half centuries ago, developed own theory of perception, whereby subjective images do not resemble the objective qualities of perceived things, but are just their abstract signs (characters). That is, any momentary perception was determined, according to Helmholtz, by the "habitual means" already formed in a given individual, due to which the constancy of the visible world is preserved.

While in this article the link between an object and a corresponding mental picture - vice versa - is rather considered as a relation of homomorphism between two non-equinumerous sets. However, to say that such homomorphic images of the same object can, in principle, exist an infinite number - this, perhaps, would be the easiest here. That's why the author is willy-nilly forced sometimes to turn in the mind's eye to the hierarchical evolution of natural systems (starting from the primary monads) in the context of the progressive development of their psyche.

And as far as the actuality around us is concerned, then final correct answer about the sought-for type of the mapping would, obviously, first of all depend here on the spatiotemporal parameters of the Universe and the comprehensive set of quantum characteristics of 5 (at least) stable elementary particles that have lied at the origins of being.

\section{Consciousness \& will}

It is methodologically convenient to distinguish 6 real levels of "Self" in a person. The primary /central/ "Self" is the main neuron of this particular hemisphere, the dominant place of which, according to vitalists, preformists and contemporary neo-Lamarckians, is defined in the embryo. In turn, yogis localize the central "Self" in the pineal gland - apparently on the basis that in lower vertebrates it plays the role of the third eye and is thus capable of intensive energy exchange. However, in our opinion, the pineal gland belongs to other important mission, namely - of the cerebral chronometer; so doesn't carry any additional loads associated with volitional activity or clairvoyance. "Self" secondary /thalamic/ is 
localized in the appropriate visual hillock. "Self" tertiary /primitive/ is built on top with the limbic system. The next level - the mammalian "Self" - including the first three sublevels, is limited to the old layers of the cortex. The fifth level - the isocortical "Self" - is characteristic of the daily activities of the vast majority of people. Finally, the sixth, highest level is the mature "Self". Each subsequent "Self" embraces all the previous ones. In terms of their functions, they differ as follows: the thalamic "Self" is responsible for practically all sensations and imprinting; the limbic "Self" does for emotions, working memory and any instinct-like manifestations; the mammalian "Self" does for elementary, one might say, animal logic; the cortical-hemispheric "Self" - for everyday thinking and long-term memory; the higher "Self" - for the formation of a worldview position, philosophical understanding of being and own place in it. That is, taking into account all of the above, in the future it would be more expedient to distinguish them from psychological positions, namely: 1) "Self" volitional; 2) "Self" sensory; 3) "Self” emotional; 4) "Self" oneiric; 5) "Self” cogitative; 6) "Self" personifical /aware, intellective/.

In the waking state, the topography of consciousness is determined by the cortical-thalamic connections that are most relevant to the individual, and the area of the covered by consciousness regions is in inverse proportion to the indicator of the distance of these zones from foremost cell /i.e. "Ego"/. Thus, consciousness is not something that is implied logically, but what is felt and experienced by the inner contemplative essence of the dominant-hemispheric "Self". In other words, there can be nothing unconscious here (i.e. on this step) per definitio. What has been said primarily concerns sensory and partly mental processes, since everything that lies below - in the brainstem or the spinal cord - is, of course, not tangible_from the inside. After all, even at the cortical level, not everyone is really feels autonomic nervous regulation, as well as stereotyped motor acts associated with the use of previously developed skills. But - let me one more time remind - all these aren't perceptions and no means certainly emotions.

The topography of consciousness in most people is not predetermined by any one organ or cell, being logically conditioned by the activity of all the main structures of the brain. Neither the higher parts of the cortex, nor the hypothalamus, nor the reticular formation, as a rule, can independently change the area of consciousness without objective reasons. And this is not anarchy at all, but just an auto-regulating system where the concept of consciousness is set axiomatically - as something that works at the moment. However, for certain people (mainly men) the central "Self" plays a decisive role in determining the sphere of consciousness. Such people are generally called strong-willed, and the rest are weak-willed. In fact there is no deep border between them. It's just that at the volitional ones, central "Self" functions much more actively, oftener taking the initiative in the distribution and ordering of nerve impulses.

True free will is always transcendental for the human mind because it can only be possessed by an auto-sufficient relic proto-energy and perhaps some the smallest but the most basic elementary particles that underlie of being. Though in everyday practice, a stable preference when choosing the sphere of consciousness of some structures of higher nervous activity over others is also usually called "firm will". That's why functional dominance in the thalamus of the reticular nucleus is manifested under the guise of the will of divine revelation; whereas a stable connection of the central "Ego" with the higher parts of the 
cerebral cortex (that are responsible for the formation of self-awareness) is mostly named rational will. It's on the latter factor that numerous methods for autogenic training and yogic improvement are based in order to achieve enlightenment. However all this of course cannot serve as an example of the manifestation of true will, because it is caused by a conscious initiative on the part of the cortex; so in the end this choice is determined by external information that the attainment of nirvana is possible and moreover it's a great indeed. Therefore, no matter how strange it may seem to some, an ordinary earthly person does not possess the factor of free will.

Latent injuries and various pathological processes developing in the central "Self" are much more dangerous than, say, hemo-blocking or even forced these cells' removal. This is especially true of strong-willed people, who, with such a turn of events, show clear signs of a severe mental illness, accompanied, moreover, by numerous somatic disorders. At the same time, for a certain (albeit usually short) period, the functions of the central "Self" can sometimes be transferred to other cells of the thalamus. And it is this phenomenon, apparently, takes place at the yogic immersing into samādhi when one of the nuclei of the reticular formation becomes dominant in their organism.

\section{OWNER \& LODGER}

In the cranial box of almost every living person and even the simplest mammal there are two different creatures - two Neighbors: the dominant (Owner) and the recessive (Lodger). The question of the possibility of their integration into an organic whole among individuals who have outstanding extra- and, sometimes, multi-sensory abilities, is still quite controversial.

It is usually not accepted to highlight separate levels of "Ego" at the Lodger, because himself is very cramped and limited in activities. However some functions are, nevertheless, fulfilled by him: so let's say, it is with his participation most of the dreams are formed; he is responsible, besides, for certain involuntary movements of the opposite side of the body; for memorizing revelations inaccessible to our active perception because of their non-entry into the sphere of consciousness; for jointly coordinated regulation of autonomic nervous system; as well as, perhaps, for some mysterious phenomena associated with the empirical rational of the chiro-doctrine, oneiromancy, divination via cards, dowsing and of course sleepwalking.

Both hemispheres of telencephalon practically belong to the Owner, but preference when choosing a program of action is, of course, given to one of them that is closer to this particular "Self". Thus, any expressions like "right-hemi's individual” and "left-hemi's man" reflect in some way his inclinations, but are not quite true to the fact in literal meaning of the word.

From a philosophical point of view, the choice between dominance and recessiveness of Neighbors is made axiomatically. Meanwhile from the standpoint of a biologist, we can talk either about genetic determination or a situational choice (related, for example, to the presence of condensed sexual heterochromatin in the corresponding progenitor ectodermal cell). But this is a purely formal alternative. In fact, of course, the type of hemispherity is set from birth; that's, there is a full genetic predestination.

Nevertheless, a locally-mosaic loss or damage of the "Y" chromosome in the cells of the dominant thalamus may well lead to incorrect differentiation in men with the development of true homosexuality. While female upper homosexuality that inevitably associated with an impudent, often even delinquent character, is largely due to the fact that genes causing such antisocial behavior were transferred to daughter from one of her parents. 
An interesting fact is that only $1 \%$ of male cells have female sex chromatin; whereas for women, respectively, the male sex chromatin is revealed 5 times as often (what is clearly discordant to statistics on the number of cases of homosexual deviations among representatives of different sexes). Hence, the conclusion about the potential belonging to this group of all uncorrectable frigid women involuntarily suggests itself. But, on the other hand, if you look at the problem through the eyes of a geneticist, the above-mentioned fact may point, moreover, to the origin of the "Y" chromosome from the "X" one.

\section{Sensations, emotions \& memory.}

As known, human memory functions on several levels, and primary (which localized in the dominant thalamus) is directly related to sensory imprinting. It's essentially the involved here monads' memory. A few seconds later, emotionally colored informational sensations can still be restored in consciousness due to reverberation of nerve impulses along the contour of thalamic nuclei or even on the overlying brain layers (if it is about, for example, the perception of speech, gestures, hieroglyphs and other difficult abstract images). Such memory can be named momentary. Generally, the limbic structures, including the hippocampus, are able to accept not only targeted orders from the thalamus, but also to catch the emotional background of any symbolically encoded messages which arrive in suitable for assimilation kind from the cortex. Wherein the hippocampus is responsible for the functioning of human working memory too.

As mentioned earlier, in the course of the historical evolution of natural systems, continuity is quite distinctly traced both in the character itself and in the way of forming sensations and emotions specific to this particular stage. But still it's most clearly seen in the example of memory, because the short-term memory of each subsequent system holds on to the mnemosubstrate of the previous one.

By the by, momentary (reverberative) memory is also apparently present at any step of the evolutionary hierarchy. But it mainly depends on the nature of the imprinting and methods the signals are transmitted, and not on the underlying structures because systems of different levels perceive the passage of time (and hence the very chronology of events) unequally.

Despite the fact that a long-term memory of chordates is based, in principle, on astrocytes, yet, if necessary, it's quite capable of appealing to similar mnemo-substance of a lower order represented, obviously, by intranuclear (but sometimes, possibly, even extracellular) proteins*. However in all people without exception, long-term memory (confined - recall just in case - to the gray matter of the cortex of both hemispheres) is generated from the working one and moreover, as a rule, during minutes of sensory lull, i.e. basically in the afternoon. At night it only is completed what was not done in daylight hours (and unless in separate short "stripes" because most of the time the brain just rests).

Although astrocytes (which, as you know, have a much shorter evolutionary "experience") store their database in analog form, and peptides - in digital, both of these functions all the same are strictly selective. Particularly, in the course of the selection of more important material, concept-proteins already present in neurons obviously play leading role, and the subcortical structures responsible for working memory are secondary. Wherein the phenomenon of total memorization is inherent only in the primary monadiform "Self" (and even then not everyone is able to get such information). 


\section{/CONCLUSION/}

The philosophical problems touched upon here, in addition to their general scientific importance, can also be of purely applied interest, since from completely another positions they reveal the nature and essence of human thinking. Which, as we know, is based precisely on long-term memory (formed, by the way, in the areas directly adjacent to specialized sensory analyzers of the cerebral hemispheres).

However, this will be in details discussed in the next author's article "Homo cogitandis - Extra-homo conscientis - Supra-homo philosophantis", which will be quite ready for publishing on the web-platform www.preprints.ru closer to winter. But still here, as a logical summary, it would not hurt to add at least a few more words about emotions. In particular, those which accompany evolutionarily significant perceptions are already emerged at the thalamic stage (i.e. simultaneously with the latters). Meanwhile ones, that require the complicity of the higher parts of telencephalon, slightly lag behind own sensations, what depends on the time it takes for the nerve impulse to pass the segment thalamus-cortexlimbus.

*However, it's not excluded that professional magicians and wonderworkers might even turn to amino-groups for the necessary information, and also to gluons, mesons, etc. (as if plunging into the truly bottomless mnemo-storage of downward systems). 